\title{
Breast Milk: Its Role in Early Development of the Immune System and Long-Term Health
}

\author{
Begüm Harmancioğlu, Seray Kabaran* \\ Department of Nutrition and Dietetics, Faculty of Health Sciences, Eastern Mediterranean University, Famagusta, Turkey \\ Email: *seray.kabaran@emu.edu.tr
}

How to cite this paper: Harmancioğlu, B. and Kabaran, S. (2019) Breast Milk: Its Role in Early Development of the Immune System and Long-Term Health. Open Journal of Obstetrics and Gynecology, 9, 458-473. https://doi.org/10.4236/ojog.2019.94045

Received: March 14, 2019

Accepted: April 9, 2019

Published: April 12, 2019

Copyright $\odot 2019$ by author(s) and Scientific Research Publishing Inc. This work is licensed under the Creative Commons Attribution International License (CC BY 4.0).

http://creativecommons.org/licenses/by/4.0/

(c) (i) Open Access

\begin{abstract}
Breast milk is the best source of nutrition that provides the energy and nutrients needed for the ideal growth and development of newborns and infants. Besides, breast milk includes various bioactive compounds, which protects infants against infectious agents and antigens and contributes to immune maturation, organ development and microbial colonization. Breast milk is dynamic; the composition of the nutrients and the content of immunological active compounds may change in each stage of lactation. During the early stages of lactation, biological and immunological active compounds provide additional support to the development of the neonatal immune system. After these stages, the composition of breast milk continues to provide appropriate energy and nutrients according to the infant needs, in order to protect neonatal immune system and maintain the development and growth of infants. Immunological maturation during the fetal life and the first months of life is provided by immunoglobulins in breast milk, which are among the most important immune protective factors and transferred to infants through breastfeeding. Due to their biological characteristics, Secretory Immunoglobulin A (SIgA) antibodies are the most important antibodies in breast milk, which provide the first defense against the antigens in the intestines of infants. In addition to antibodies, enzymes, including active leukocytes, cytokines, oligosaccharides, lactoferrin, lysozyme and lactoperoxidase, as well as biological and immunological factors, such as hormones, growth factors, bioactive peptides, nucleotides and fatty acids are transferred to infants through breastfeeding. There is now a growing body of evidence suggesting that breastfeeding protects infants against many infections such as gastrointestinal system and respiratory tract infections, strengthens immune system and provides protective effects against allergic and autoimmune diseases in later life.
\end{abstract}

\section{Keywords}

Breast Milk, Breast Milk Immunology, Neonatal Immune System, Immune-Mediated Diseases 


\section{Introduction}

Breast milk is a unique source of nutrition that meets the nutritional and physiological needs of the newborn. Breast milk is considered as the "gold standard" for infant nutrition [1]. World Health Organization (WHO) and UNICEF recommendations on breastfeeding are as follows: initiation of breastfeeding within the first hour after the birth; exclusive breastfeeding for the first six months; and continued breastfeeding for two years or more together with safe and appropriate complementary foods [2] [3].

Breast milk contains all the nutrients required for the growth and development of infants. Besides, breast milk contains various specific and unspecific compounds that protect infants against infectious agents and antigens and support the development of neonatal immune system, which is influenced by maternal immunity transferred to newborns through placenta and breast milk [4]-[9]. Immune compounds in breast milk include anti-infectious and anti-inflammatory agents, active leukocytes (neutrophils, T-cells and macrophages), and immunomodulators [10]. In addition, antimicrobial factors in breast milk provide resistance against digestive enzymes, protect mucosal surfaces and eliminate bacteria without causing inflammatory reactions [5].

A significant number of studies provide evidences for the benefits of breast milk for infants. Protective effects of breastfeeding on gastrointestinal system and respiratory tract infections have already been indicated. However, it is stated that breast milk does not only provide passive protection, but can also directly change the immunological development of the infants. Various studies found that breastfeeding promotes cognitive development, decreases inflammation and reduces the risk of developing allergic and chronic diseases such as obesity, Type 1 and Type 2 diabetes and cardiovascular diseases later in life [10]-[17].

Some of the epidemiological studies found that formula feeding increases the risk for diseases compared to breastfeeding [15] [18]. Consequently, it is considered that breast milk has the most protective and effective immunomodulatory activities among the other nutrients and infant formula. However, how some of the specific factors involved in breast milk contribute to both innate and adaptive immune functions of neonates and infants is still being investigated [4] [14] [19].

\section{Neonatal Immune System, Biological and Immunological Factors of Breast Milk}

Breast milk is a source that is known to be the richest in terms of immunomodulation [14]. It has three stages, namely colostrum (the first milk secreted in the first five days postpartum), transitional milk (secreted between 6 - 15 days postpartum) and mature milk (secreted after $16^{\text {th }}$ day postpartum). Nutrients and the composition of breast milk changes in each stage of lactation. For example, colostrum is secreted in low quantities but is highly important for infants since it is rich in immunologic and developmental factors. Transitional milk is secreted 
more than the colostrum in order to provide infant's development and nutrient requirements. Besides, compared to colostrum, transitional milk contains lower amounts of immunoglobulins and protein but higher amounts of energy, lactose and fat. Finally, mature milk is fully matured in terms of nutrients [1] [10] [20].

Infectious diseases, including upper and lower respiratory tract infections, otitis media, sepsis, meningitis and gastroenteritis, may result with morbidity and mortality during the infants first months of life [5]. Similar to other systems found in the body, immune system of newborn is not fully functional at birth and has not yet developed. With parturition, newborns move from the sterile environment in the uterus to the world, which is full of pathogen microorganisms [21]. Incomplete physical and chemical barriers, innate weak effect or cell function, limited and delayed Secretory Immunoglobulin A (SIgA) production, and insufficient anti-inflammatory mechanisms of the respiratory and gastrointestinal tracts are among the important developmental immune deficiencies at birth [4].

Postpartum period is extremely important for the maturation of neonatal immune system. Thymus is the main organ of the central immune system and the process is characterized by the balance between Th1/Th2 responses [22]. Immunological maturation during the fetal life and the first months of life is maintained by the transfer of some of the maternal protective proteins to the infant via breastfeeding. Among these, antibodies are the most protective proteins. For example, transplacental transfer of antibodies to the fetus, anti-infective resistance factors in the amniotic fluid and transfer of immunological factors via colostrum and breast milk strengthen the immune system of infants [6] [7] [21]. Immunoglobulin $\mathrm{G}(\mathrm{IgG})$, which is the dominant antibody at birth, is detected in the serum and non-mucosal tissues, and protects newborns against pathogens and toxins. In addition, low amounts of IgM, IgE, IgD and IgA are also found in the serum. Although the maternal antibodies decrease gradually after birth, neonates and infants may benefit from such protective factors, thanks to breast milk [7] [23] [24].

The composition of breast milk changes parallel to the development of the infants. In addition to antibodies, breast milk contains living cells and biologically and immunologically active agents, including leukocytes (polymorphonuclear neutrophils, monocytes/macrophages, lymphocytes), cytokines, inflammatory mediators, signaling molecules, hormones, growth factors, soluble receptors, oligosaccharides, polyunsaturated fatty acids and various microbiomes [25] [26] [27]. All these agents interact with each other and the mucous membranes of the digestive and upper respiratory tracts of infants, providing passive immunity and strengthening the immune system [5].

\subsection{SIgA}

Immunoglobulins in breast milk are the best immune protectors discovered so far. Although all immunoglobulin isotypes are found in colostrum and breast 
milk, SIgA is considered as the most important immunoglobulin due to its biological characteristics. IgM and IgG are the other most found immunoglobulins in breast milk after IgA. Colostrum contains $12 \mathrm{mg} / \mathrm{ml}$ SIgA, whereas the amount of SIgA in mature milk is $1 \mathrm{mg} / \mathrm{ml}$ [28] [29]. Besides, the amount of immunoglobulins in bovine milk and infant formula is less than the breast milk [30]. Daily intakes of SIgA by exclusively breastfed infants are about $0.5-1.0$ $\mathrm{g} / \mathrm{kg}[28]$.

Breast milk IgA is synthesized by B cells that migrate from the small intestines of the mother to mammary glands by CCL28 chemokine. IgA is synthesized in a dimeric form and is attached to secretory component [8] [31]. SIgA antibodies in breast milk play an important role on mucous membrane defense. These antibodies provide the first protection for infant against the foreign antigens in the intestines. SIgA molecules provide protection against mucosal pathogens by preventing pathogenic microorganisms, toxins, bacteria (Escherichia coli, Vibrio cholerae, Campylobacter, Shigella, Giardia lamblia, Haemophilus influenzae, Clostridium difficile, and Streptococcus pneumoniae), viruses (Rotavirus, CMV, Cytomegalovirus, HIV, Influenza virus, respiratory syncytial virus), fungus (Candida albicans) and antigens such as lipopolysaccharide (LPS) from attaching to epithelial cells without triggering inflammatory reactions(immune exclusion). Also, since SIgA is relatively resistant against proteolysis, it maintains protection against the pathogens in the gastrointestinal tracts. In this sense, SIgA maintains immune defense for infants by preventing bacterial colonies and the translocation across the mucosal barrier [5] [6] [8] [28] [31].

\subsection{Breast Milk Cells}

Breast milk contains various living cells. Epithelial cell adhesion molecules $($ EpCAM+) are the most common type of cells in the breast milk. Moreover, breast milk contains leukocytes. Mature milk contains more leukocytes than colostrum. Colostrum contains nearly $5 \times 10^{6}$ cells per ml. As breast milk matures, this amount decreases tenfold in the milk [23]. On the other hand, the percentage of neutrophils in leukocytes is approximately $80 \%$, whereas the percentages for macrophages and immune cells such as lymphocytes are $15 \%$ and $4 \%$, respectively [31]. There are various types of lymphocytes in breast milk. Nearly $83 \%$ of lymphocytes in the breast milkis T (CD3+) cells, $11 \%$ is gdT cells, $3 \%$ $4 \%$ is CD16+ NK cells and 2\% is B cells [8] [31]. In addition, breast milk contains stem cells. Although their roles in neonatal immune system are not completely understood, epithelial and stem cells protect infants against potential pathogens and play a key role in neonatal protection [25].

\subsection{Cytokines and Chemokines}

Protection against microorganisms, including bacteria and viruses, is provided by either innate or acquired immunity. This protection is provided by protein hormones, called cytokines [10]. Cytokines are glycoproteins that have various functions and that are crucial for the activation of immune system and intercel- 
lular communication [32]. Besides, cytokines in breast milk have immunomodulatory effects on the development and maturation of phagocytic cells and lymphocytes, which play a role in the development of immune response against allergic reactions in infants [10]. By targeting cells in breast milk, cytokines contribute to the development, differentiation and production of immunoglobulins with the help of B cells, and promotes thymus proliferation [10]. Cytokines in breast milk include tumor necrosis factor- $\alpha$ (TNF- $\alpha$ ), transforming growth factor- $\beta$ (TGF- $\beta$ ), interferon- $\gamma$ (IFN- $\gamma$ ), interleukin- $\beta$ (IL- $1 \beta$ ), IL-2, IL-4, IL-6, IL-7, IL-8, IL-10, IL-12, IL-13, IL-16 and IL-18 [31]. Cytokines may stimulate IgA production by peripheral blood lymphocytes [33]. Among these cytokines, TGF- $\beta$, IL-6, IL-7 and IL-10 are crucial for the development and differentiation of cells that produce IgA, whereas IL-6 is important for the development of mucosal immunity in infants [34]. IL-10 is an anti-inflammatory and immune regulatory cytokine that is found both in water and fat layers in high concentrations. Similar to IL-10, TGF- $\beta$ has important immunomodulatory characteristics, including the stimulation of the maturation of immune system and plays an important role in preventing allergic diseases in infants by suppressing $\mathrm{T}$ cells (Th1 and Th2) [5] [35]. Additionally, cytokines in breast milk helps the transfer of leukocytes to the breast milk and the intestinal epithelium of infants [32].

Chemokines are small chemotactic cytokines that have the target cell selectivity to activate leukocytes. CXC chemokines have the ability to activate neutrophils where as CC chemokines do not have such an ability. CXC chemokines are found in breast milk in large amounts and play a vital role in defense against bacterial and viral infections [5] [10].

\subsection{Oligosaccharides, Prebiotics and Probiotics}

Breast milk contains various complex biological agents that support the intestinal microbiota of infant, including saccharides (mostly oligosaccharides), amino acids, vitamins, minerals and other nutrients [36]. Oligosaccharides are important nutrients in breast milk and are antimicrobial factors, called glycans. Glycans are synthesized from lactose in mammary gland. By showing resistance gastrointestinal digestion, glycans enter the infant intestines as the first prebiotics. Consequently, oligosaccharides help to protect infant microbiome by attaching to pathogens [4] [36] [37].

Oligosaccharides are the third largest compounds in breast milk after lactose and fat and constitute an important share of carbohydrates. The amount of oligosaccharides in mature milk is approximately $12.9 \mathrm{~g} / \mathrm{L}$ whereas colostrum contains $20.9 \mathrm{~g} / \mathrm{L}$ of oligosaccharides [28]. N-acetyl glucosamine that contains oligosaccharides has been defined as "bifidus factor" many years ago. N-acetyl glucosamine is a monosaccharide that uses as a substrate for intestinal bifidobacteria, which is responsible for bifidogenic activity of oligosaccharides in breast milk. Bifidobacteria species in breast milk are the main users of oligosaccharides in gastrointestinal system and play a vital role for microbiota of breastfed 
infants and in maintaining the general health of infants [36]. Breast milk oligosaccharides have inhibitory effects on the adhesion of pathogenic microorganisms to the intestinal mucosa, the growth of pathogens through the production of bacteriocins and organic acids and the expression of genes that play a role in inflammation. Besides, bifidobacteria species contribute to intestinal health by indirectly increasing the production of short-chain fatty acids (SCFAs) [37] [38] [39].

Breast milk oligosaccharides and microbiota are the two fundamental milk components that affect the infant's intestinal microbiota, and consequently, the development of the immune system. Colostrum and mature milk are continuous sources of commensal and potentially probiotic bacteria for the infant's intestine and intramammary breast milk has traditionally been considered as sterile [5]. So that, colostrum and mature milk have prebiotic and probiotic characteristics that modulate intestinal microbiota, including acquiring new bacterial species and providing a permissive environment to facilitate sustainable colonization [40] [41]. Breast milk microbiota may originate from the maternal intestine, breast tissue or infant oral cavity. Depending on the source of bacteria, various factors may influence to the formation of breast milk microbiota [40]. Breast milk is an important source for the infants' intestines since an infant that consumes approximately $800 \mathrm{~mL} /$ day of breast milk digests $1 \times 10^{5}$ and $1 \times 10^{7}$ bacteria per day [5]. Degradation of the prebiotic and probiotic properties of the breast milk alters the development of the intestinal microbiota of infants, which, in turn, may be the underlying mechanisms of various chronic diseases including allergy and asthma [40] [41]. Without prebiotic or probiotic supplementations, intestinal microbiota of formula-fed infants lacks Bifidobacteria species. According to the previous studies, it is stated that the breastfed infants consumed more stable and uniform oligosaccharides than the formula-fed infants [42]. Additionally, it has been reported that there is an association between breastfeeding (directly with breast or bottle-feeding) and microbiota composition of breast milk. Feeding the infant with expressed breast milk decreases the amount of bifidobacteria and leads to exposure to pathogens [40]. Another study found that the administration of a follow-on formula with Lactobacillus fermentum CECT5716 to the infants between the ages of 6 and 12 months may be helpful to prevent gastrointestinal and upper respiratory infections [43].

\subsection{Lactoferrin}

Lactoferrin is one of the main whey proteins of breast milk that helps the absorption of iron and has the killer effects of microbes in the intestinal mucosa of infants. The amounts of lactoferrin in $100 \mathrm{ml}$ of colostrum and mature milk are 7 gram and $0.1-0.3$ gram, respectively. Lactoferrin concentrations in breast milk are controlled by reproductive hormones, such as, prolactin and estrogen [8] [9] [31]. As an iron-binding protein, lactoferrin has bacteriostatic effects on infant's intestinal mucosa. Lactoferrin inhibits the use of iron, which is necessary for the 
growth of various pathogens by linking the iron in the digestive system of infants [5] [8] [14]. By preventing bacteria adhering to the intestinal cells, lactoferrin has a direct cytotoxic effect against Gram-positive and Gram-negative bacteria (Listeria, E. coli, Salmonella, and Campylobacter), fungi and viruses (HIV-1) [5]. Lactoferrin also inhibits the production of proinflammatory cytokines (IL-6, IL-8 TNF- $\alpha$ and IL-1 $\beta$ ) and inflammatory mediators, stimulates the production and maturation of lymphocytes, and consequently, contributes to the development of the infant's immune system. A part of the lactoferrin activity is attributed to the formation of lactoferricin, which is a powerful peptide that is formed during the digestion of lactoferrin [4] [5] [8].

\subsection{Lysozyme and Lactoperoxidase}

Lysozyme is an important part of the whey protein fraction and it has the highest concentrations in colostrum. The amount of lysozyme in breast milk is approximately 10 micrograms $(\mu \mathrm{g}) / \mathrm{dL}$, which is 3000 times higher than the amount in bovine milk. Together with SIgA, lactoferrin and other antimicrobial compounds, lysozyme plays a vital role for the immune defense of infants [8] [14] [31] [44]. Lactoferrin removes the LPS from the outer membrane of the cells and allows lysozyme to enter the cell and degrade the internal proteoglycan matrix of the membrane. Besides, lysozyme has also antiviral activities [5].

In addition to lysozyme, breast milk also contains lactoperoxidase enzyme, which has antibacterial properties. In the presence of hydrogen peroxide, lactoperoxidase forms hypothiocyanite, which may kill both Gram-positive and Gram-negative bacteria, and catalyzes the oxidation of thiocyanate. Consequently, lactoperoxidase provides defense against the bacteria in the mouth and upper gastrointestinal system [5] [8].

\subsection{Hormones, Growth Factors and Bioactive Peptides}

Cortisol, gender hormones (estrogen, progesterone), thyroid hormones, peptides related with parathyroid hormone, adrenocorticotropic hormones, gastrointestinal regulatory peptides (gastrin, neurotensin, peptide YY, somatostatin, etc.), erythropoietin, gonadotropin, insulin, leptin, adiponectin, hormones related with hypothalamus ( $\mathrm{GnRH}, \mathrm{GH}$, prolactin, TSH, etc.), growth factors (epidermal growth factor [EGF], insulin-like growth factor [IGF] I and II, etc.), $\alpha$-lactoalbumin, $\beta$-lactoglobulin and lactoferrin are the hormones, growth factors and partially digested peptides in breast milk, which are also bioactive compounds of the breast milk [8] [45]. IGF and leptin in breast milk are the hormones that may module the immune system of intestinal mucosa by regulating cytokine expression and other signal pathways [41]. In addition, peptide hormones, such as ghrelin and adiponectin involve in the regulation of appetite, energy balance, cell differentiation, and contribute to the development in the neonatal period [46]. IGF I and II are bound to IGF-binding proteins, which helps to protect them against digestion and modulate the interaction with the intestinal receptors. 
IGFs may show bioactivity after being bounded to enterocytes [44].

\subsection{Nucleotides}

Nucleotides or nucleic acids comprise approximately $15 \%-20 \%$ of the non-protein nitrogen content in breast milk. In vitro and in vivo studies found that nucleotides increased iron absorption, bifidobacteria growth, development and repair of gastrointestinal mucosa, NK cell activity and IL-2 production [5] [47]. Besides, nucleotides support Th1 response and regulate differentiation and maturation of $\mathrm{T}$ and $\mathrm{B}$ cells [8] [31].

\subsection{Fatty Acids}

Polyunsaturated fatty acids (PFAs) in breast milk, including omega-3 fatty acid, Docosahexaenoic acid (DHA) and Eicosapentaenoic acid (EPA) have anti-inflammatory effects on chronic inflammatory diseases, such as asthma [27]. Nevertheless, a high arachidonic acid to EPA ratio in breast milk may be related with a higher risk of allergic disease and atopy [26]. Recently, acetate, butyrate and propionate, which are among the SCFAs have been reported as the agents of allergic inflammation. SCFAs are the first metabolites produced by the intestinal microbiota of the newborns and their synthesis increase after the birth [27].

\section{Possible Impacts of Breast Milk on Immune-Mediated Diseases}

\subsection{Allergic Diseases}

Although several studies in the literature show that breast milk maintains defense against infections and allergic diseases, the results of the studies investigating the effects of breastfeeding on atopic diseases are conflicting. In some studies, it is claimed that breastfeeding has a protective effect against the atopic diseases due to the duration of breastfeeding, whereas some of them did not make a certain decision on this issue. However, in a small number of studies, it is suggested that prolonged breastfeeding may increase the risk of the development of atopic diseases [48] [49] [50]. Systematic reviews and meta-analysis reveal that breastfeeding protects against atopic dermatitis, asthma, allergic rhinitis and allergy against bovine milk during the early childhood period, so that they suggest breastfeeding in order to prevent the development of allergic diseases [51] [52] [53].

\subsection{Asthma}

Alteration of the microbiota during the neonatal period due to antibiotic usage, cesarean delivery or formula feeding may contribute to the development of asthma in infants and children [54]. However, there is no study showing a direct relationship between breast milk microbiota and the development of asthma and allergy. Oligosaccharides in breast milk affect lung mucosal immunity in order to maintain protection against asthma by interacting with the respiratory tract 
epithelium, immune cells, potential pathogens or existing microbes [40] [55]. Although the underlying mechanisms are not fully understood, studies on animals or humans suggest that prebiotic or oligosaccharides supplementation may be protective against allergy and asthma [56] [57]. For example, one of the studies found that prebiotic supplementation with galacto-oligosaccharide (GOS) and fructo-oligosaccharide (GOS) decreased the risk of asthma in infants at high risk of allergy [58].

\subsection{Food Allergy}

In the literature, there are contradictions between studies evaluating the relationship between breastfeeding and food allergies [28]. One of the studies found that exclusive breastfeeding for the first 4 months of life decreased the risk of developing food allergy in children at high risk [59], whereas another study found that long-term ( $\geq 9$ months) exclusive breastfeeding increased the risk of food allergy in childhood [60]. On the other hand, a meta-analysis found no statistically significant relationship between breastfeeding and the development of food allergy [53]. The presence of atopy in mothers may influence the immunology of breast milk. One of the studies found that IgA concentrations in the breast milk of atopic mothers were lower compared to breast milk of non-atopic mothers, but this was not related to the development of food allergy in children [61]. However, in another study, it was reported that lower SIgA levels in breast milk increased the risk of cow's milk allergy in infants [62]. A study about food allergy found that the concentration of the TGF $\beta$-1in colostrum of the mothers of infants with IgE-mediated cow's milk allergy was lower than the colostrum of mothers of infants with non-IgE mediated cow's milk allergy [63]. In addition, fatty acids, which are among the immunological components of the breast milk may play a role in food allergy. Thijs et al. [64] found an inverse association between omega- 3 and rumenic fatty acid content of breast milk and the food sensitization in the first year of life. Besides, oligosaccharides in the breast milk may be protective against cow's milk allergy due to its positive effects on intestinal microbiome [48].

\subsection{Eczema}

A comprehensive systematic review and meta-analysis found that children under 2 years of age who were exclusively breastfed for more than 4 months had lower risks of eczema development; however this impact did not continue after 2 years of age [53]. IL-1 $\beta$, IL-6, IL-10 and TGF $\beta$ - 1 in breast milk increases the production of IgA and may be protective against atopic dermatitis (eczema) and cow's milk allergy. However, there are some studies showing that high IgA concentrations in breast milk have both protective [65] and unprotective [66] effects against the development of eczema. Another study demonstrated that low TGF $\beta$ - 1 concentrations in breast milk might be related with the development of eczema [67]. However, Munblit et al. [68] reported no relationship between IL-2, 
IL-4, IL-5, IL-10, IL-12 and IFN- $\gamma$ found in breast milk and eczema or food allergy.

\subsection{Autoimmune Diseases}

In the literature, there are some studies dealing with the role of breast feeding on the development of the diseases in which breastfeeding plays a central role in the pathogenesis of inflammation. Many components found in breast milk affect the development and function of the immune system, and thus, provide protective effects against allergic and autoimmune diseases [22].

Type 1 diabetes is a disease usually caused by genetic factors and immune system disorders. Although there is no direct evidence for the protective effects of breastfeeding against Type 1 diabetes, breastfeeding may indirectly prevent Type 1 diabetes through its effects, such as lower risk for diabetogenic infection, healthy intestinal microbiota and maturation of newborn's intestines [22]. Cardwell et al. [69] reported that exclusive breastfeeding in infants who were genetically at risk of developing Type 1 diabetes for the first 2 weeks immediately after birth or for more than 3 months reduced the risk of developing Type 1 diabetes by $15 \%-30 \%$ in childhood. Nevertheless, the protective effects of breastfeeding against Type 1 diabetes are controversial [23].

It is known that breastfeeding reduces the risk of developing obesity and metabolic syndrome in later life. Studies suggest that breastfeeding reduces the risk of developing Type 2 diabetes in childhood by $24 \%$. For example, a study showed that children with normal weight had a history of breastfeeding for more than 6 months compared to their Type 2 diabetic obese peers [70].

Juvenile idiopathic arthritis (JIA) is a chronic rheumatic disease that is highly common in childhood. Although its pathogenesis and etiology is not fully understood, genetic factors, immune mechanisms and environmental factors have been reported to play a role in the development of JIA. The protective effects of breastfeeding on the development of JIA are not certain. For example, a prospective cohort study found that breastfeeding (both exclusive and in total) for more than 4 months may have a protective effect against the development of JIA. However, children who were breastfed for less than 4 months were reported to have a higher risk of JIA development [71]. Another study found that breastfeeding had no impact on the development of JIA [72].

Celiac disease is a systemic immune-mediated disease and caused by the ingestion of gluten-containing grains in individuals genetically sensitive to gluten. The relationship between breastfeeding and celiac disease may stem from the delay of gluten consumption due to breastfeeding [73]. However, it is not certain yet whether breast milk actually reduces the risk of developing celiac disease or delays the diagnosis of celiac disease [22].

Inflammatory bowel diseases (ulcerative colitis and Crohn's) are the result of impaired immune response against pathogens and chronic inflammation of the gastrointestinal system. Although its pathogenesis is not known, family history is 
the main risk factor for the development of inflammatory bowel diseases. The findings on the relationship between breastfeeding and inflammatory bowel diseases are controversial. In the literature, there are studies suggesting that breast feeding has protective effects against inflammatory bowel diseases [74], whereas others suggest no relationship between breastfeeding and inflammatory bowel diseases [75].

In addition to these diseases, some of the epidemiological studies found that breastfeeding decreases the risk of immune-mediated diseases, such as necrotizing enterocolitis, obesity, atherosclerosis, respiratory tract infection, rheumatoid arthritis, and some of the childhood cancers, including lymphomas and leukemia [8].

\section{Conclusion}

Breast milk is the ideal source for optimal growth and development of infants and provides numerous benefits to the infant. Besides, breast milk is also rich in many immunological factors that provide the first adaptive immunity in the gastrointestinal tract of infants. Breastfeeding not only provides the ideal nutrient composition for the newborn but also maintains an excellent immunological integration between the mother and infant. The composition of immune components of breast milk may vary due to maternal factors. Accordingly, although all mechanisms for the association between breastfeeding and the prevention of development of allergic diseases have not been fully understood, it has been demonstrated that breast milk has beneficial effects on some immune-mediated diseases. Recent technological developments continue to reveal possible mechanisms for better identification of breast milk composition and beneficial effects of breast milk on both short and long-term health. Therefore, prospective cohort studies are thought to shed better light on the important factors, which provide key roles in the development of immune-related diseases.

\section{Approved}

The design and conduct of the work was performed by all the authors. The manuscript has been written, read, and approved by all the authors. The material has not been previously published, in whole or in part, and it also is not under consideration for publication elsewhere.

\section{Conflicts of Interest}

The authors declare no conflicts of interest regarding the publication of this paper.

\section{References}

[1] Garwolińska, D., Namieśnik, J., Kot-Wasik, A. and Hewelt-Belka, W. (2018) Chemistry of Human Breast Milk-A Comprehensive Review of the Composition and Role of Milk Metabolites in Child Development. Journal of Agriculture Food and Chemistry, 66, 11881-11896. https://doi.org/10.1021/acs.jafc.8b04031

[2] World Health Organization. Exclusive Breastfeeding for Optimal Growth, Devel- 
opment and Health of Infants. e-Library of Evidence for Nutrition Actions (eLENA). https://www.who.int/elena/titles/exclusive_breastfeeding/en/

[3] UNICEF (2015) Breastfeeding. UNICEF for Every Child. https://www.unicef.org/nutrition/index_24824.html

[4] Cacho, N.T. and Lawrence, R.M. (2017) Innate Immunity and Breast Milk. Frontiers in Immunology, 8, 584. https://doi.org/10.3389/fimmu.2017.00584

[5] Palmeira, P. and Carneiro-Sampaio, M. (2016) Immunology of Breast Milk. Revista da Associação Médica Brasileira, 62, 584-593.

https://doi.org/10.1590/1806-9282.62.06.584

[6] Hanson, L.A., Korotkova, M., Lundin, S., Haversen, L., Silfverdal, S.A. and Mattsby-Baltzer, I. (2003) The Transfer of Immunity from Mother to Child. Annals of the New York Academy of Sciences, 987, 199-206. https://doi.org/10.1111/j.1749-6632.2003.tb06049.x

[7] Jennewein, M.F., Abu-Raya, B., Jiang, Y., Alter, G. and Marchant, A. (2017) Transfer of Maternal Immunity and Programming of the Newborn Immune System. Seminars in Immunopathology, 39, 605-613. https://doi.org/10.1007/s00281-017-0653-x

[8] Blewett, H.J.H., Cicalo, M.C., Holland, C.D. and Field, C.J. (2008) The Immunological Components of Breast Milk. Advances in Food and Nutrition Research, 54, 45-80. https://doi.org/10.1016/S1043-4526(07)00002-2

[9] Walker, A. (2010) Breast Milk as the Gold Standard for Protective Nutrients. The Journal of Pediatrics, 156, S3-S7. https://doi.org/10.1016/j.jpeds.2009.11.021

[10] Ballard, O. and Morrow, A.L. (2013). Breast Milk Composition: Nutrients and Bioactive Factors. Pediatric Clinics, 60, 49-74.

[11] Goldman, A.S. (2018) Future Research in the Immune System of Breast Milk. The Journal of Pediatrics, 206, 274-279. https://doi.org/10.1016/j.jpeds.2018.11.024

[12] Lönnerdal, B., Erdmann, P., Thakkar, S.K., Sauser, J. and Destaillats, F. (2017) Longitudinal Evolution of True Protein, Amino Acids and Bioactive Proteins in Breast Milk: A Developmental Perspective. The Journal of Nutritional Biochemistry, 41, 1-11. https://doi.org/10.1016/j.jnutbio.2016.06.001

[13] Edmond, K.M., Zandoh, C., Quigley, M.A., Amenga-Etego, S., Owusu-Agyei, S. and Kirkwood, B.R. (2006) Delayed Breastfeeding Initiation Increases Risk of Neonatal Mortality. Pediatrics, 117, e380-e386. https://doi.org/10.1542/peds.2005-1496

[14] Levy, O. (2007) Innate Immunity of the Newborn: Basic Mechanisms and Clinical Correlates. Nature Reviews Immunology, 7, 379-390.

https://doi.org/10.1038/nri2075

[15] Wall, G. (2013) Outcomes of Breastfeeding versus Formula Feeding. https://www.evergreenperinataleducation.com/upload/OutcomesofBreastfeeding_N ov2013.pdf

[16] Cunningham, A.S., Jelliffe, D.B. and Jelliffe, E.P. (1991) Breast-Feeding and Health in the 1980s: A Global Epidemiologic Review. The Journal of Pediatrics, 118, 659-666. https://doi.org/10.1016/S0022-3476(05)80023-X

[17] Stuebe, A. (2009) The Risks of Not Breastfeeding for Mothers and Infants. Reviews in Obstetrics and Gynecology, 2, 222-231.

[18] Khadivzadeh, T. and Parsai, S. (2004) Effect of Exclusive Breastfeeding and Complementary Feeding on Infant Growth and Morbidity. Journal of Eastern Mediterranean Health, 10, 289-294.

[19] Alexander, K.L., Targan, S.R. and Elson III, C.O. (2014) Microbiota Activation and 
Regulation of Innate and Adaptive Immunity. Immunological Reviews, 260, 206-220. https://doi.org/10.1111/imr.12180

[20] Kleinman, R.E. and Greer, F.R. (2014) Micronutrients and Macronutrients. In: Pediatric Nutrition, 7th Edition, American Academy of Pediatrics, Committee on $\mathrm{Nu}$ trition, Itasca, IL, 357-568.

[21] Tregonin, J. Neonatal Immunology. https://www.immunology.org/public-information/bitesized-immunology/immunedevelopment/neonatal-immunology

[22] Vieira Borba, V., Sharif, K. and Shoenfeld, Y. (2018) Breastfeeding and Autoimmunity: Programing Health from the Beginning. American Journal of Reproductive Immunology, 79, e12778. https://doi.org/10.1111/aji.12778

[23] Jackson, K.M. and Nazar, A.M. (2006) Breastfeeding, the Immune Response, and Long-Term Health. The Journal of the American Osteopathic Association, 106, 203-207.

[24] Roopenian, D.C. and Akilesh, S. (2007) FcRn: The Neonatal Fc Receptor Comes of Age. Nature Reviews Immunology, 7, 715-725. https://doi.org/10.1038/nri2155

[25] Hoyt, A.E., Medico, T. and Commins, S.P. (2015) Breast Milk and Food Allergy: Connections and Current Recommendations. Pediatric Clinics, 62, 1493-1507.

[26] Oddy, W.H. (2017) Breastfeeding, Childhood Asthma, and Allergic Disease. Annals of Nutrition and Metabolism, 70, 26-36. https://doi.org/10.1159/000457920

[27] Munblit, D., Peroni, D., Boix-Amorós, A., Hsu, P., Land, B. and Gay, M. (2017) Human Milk and Allergic Diseases: An Unsolved Puzzle. Nutrients, 9, 894. https://doi.org/10.3390/nu9080894

[28] Andreas, N.J., Kampmann, B. and Le-Doare, K.M. (2015) Human Breast Milk: A Review on Its Composition and Bioactivity. Early Human Development, 91, 629-635. https://doi.org/10.1016/j.earlhumdev.2015.08.013

[29] Goldman, A.S., Garza, C., Nichols, B.L. and Goldblum, R.M. (1982) Immunologic Factors in Breast Milk during the First Year of Lactation. The Journal of Pediatrics, 100, 563-567. https://doi.org/10.1016/S0022-3476(82)80753-1

[30] Chatterton, D.E., Nguyen, D.N., Bering, S.B. and Sangild, P.T. (2013) Anti-Inflammatory Mechanisms of Bioactive Milk Proteins in the Intestine of Newborns. The International Journal of Biochemistry and Cell Biology, 45, 1730-1747. https://doi.org/10.1016/j.biocel.2013.04.028

[31] Lepage, P. and Van de Perre, P. (2012) The Immune System of Breast Milk: Antimicrobial and Anti-Inflammatory Properties. In: Kourtis, A. and Bulterys, M., Eds., Human Immunodeficiency Virus Type $1(H I V-1)$ and Breastfeeding. Advances in Experimental Medicine and Biology, Springer, New York, 121-137. https://doi.org/10.1007/978-1-4614-2251-8_9

[32] Ustundag, B., Yilmaz, E., Dogan, Y., Akarsu, S., Canatan, H. and Halifeoglu, I. (2005) Levels of Cytokines (IL-1 $\beta$, IL-2, IL-6, IL-8, TNF- $\alpha$ ) and Trace Elements (Zn, $\mathrm{Cu})$ in Breast Milk from Mothers of Preterm and Term Infants. Mediators of Inflammation, 2005, 331-336. https://doi.org/10.1155/MI.2005.331

[33] Brandtzaeg, P. (2010) The Mucosal Immune System and Its Integration with the Mammary Glands. The Journal of Pediatrics, 156, S8-S15.

https://doi.org/10.1016/j.jpeds.2009.11.014

[34] Brandtzaeg, P. (2003) Mucosal Immunity: Integration between Mother and the Breast-Fed Infant. Vaccine, 21, 3382-3388. https://doi.org/10.1016/S0264-410X(03)00338-4

[35] Verhasselt, V. (2010) Neonatal Tolerance under Breastfeeding Influence: The Pres- 
ence of Allergen and Transforming Growth Factor- $\beta$ in Breast Milk Protects the Progeny from Allergic Asthma. The Journal of Pediatrics, 156, S16-S20. https://doi.org/10.1016/j.jpeds.2009.11.015

[36] Musilova, S., Rada, V., Vlkova, E. and Bunesova, V. (2014) Beneficial Effects of Breast Milk Oligosaccharides on Gut Microbiota. Beneficial Microbes, 5, 273-283. https://doi.org/10.3920/BM2013.0080

[37] Plaza-Díaz, J., Fontana, L. and Gil, A. (2018) Breast Milk Oligosaccharides and Immune System Development. Nutrients, 10, 1038. https://doi.org/10.3390/nu10081038

[38] Bode, L. (2012) Human Milk Oligosaccharides: Every Baby Needs a Sugar Mama. Glycobiology, 22, 1147-1162. https://doi.org/10.1093/glycob/cws074

[39] Morozov, V., Hansman, G., Hanisch, F.G., Schroten, H. and Kunz, C. (2018) Human Milk Oligosaccharides as Promising Antivirals. Molecular Nutrition and Food Research, 62, Article ID: 1700679. https://doi.org/10.1002/mnfr.201700679

[40] Moossavi, S., Miliku, K., Sepehri, S., Khafipour, E. and Azad, M. (2018) The Prebiotic and Probiotic Properties of Human Milk: Implications for Infant Immune Development and Pediatric Asthma. Frontiers in Pediatrics, 6, 197. https://doi.org/10.3389/fped.2018.00197

[41] Gomez-Gallego, C., Garcia-Mantrana, I., Salminen, S. and Collado, M.C. (2016) The Human Milk Microbiome and Factors Influencing Its Composition and Activity. Seminars Fetal and Neonatal Medicine, 21, 400-405. https://doi.org/10.1016/j.siny.2016.05.003

[42] Martin, C.R., Ling, P.R. and Blackburn, G.L. (2016) Review of Infant Feeding: Key Features of Breast Milk and Infant Formula. Nutrients, 8, 279. https://doi.org/10.3390/nu8050279

[43] Maldonado, J., Cañabate, F., Sempere, L., Vela, F., Sánchez, A.R. and Narbona, E. (2012) Breast Milk Probiotic Lactobacillus Fermentum CECT5716 Reduces the Incidence of Gastrointestinal and Upper Respiratory Tract Infections in Infants. Journal of Pediatric Gastroenterology and Nutrition, 54, 55-61. https://doi.org/10.1097/MPG.0b013e3182333f18

[44] Lönnerdal, B. (2010) Bioactive Proteins in Breast Milk: Mechanisms of Action. The Journal of Pediatrics, 156, S26-S30. https://doi.org/10.1016/j.jpeds.2009.11.017

[45] Lawrence, R.M. (2007) Human Breast Milk: Current Concepts of Immunology and Infectious Diseases. Current Problems in Pediatric and Adolescent Health Care, 37, 7-36. https://doi.org/10.1016/j.cppeds.2006.10.002

[46] Wada, Y. and Lönnerdal, B. (2014) Bioactive Peptides Derived from Breast Milk Proteins-Mechanisms of Action. The Journal of Nutritional Biochemistry, 25, 503-514. https://doi.org/10.1016/j.jnutbio.2013.10.012

[47] Pickering, L.K., Granoff, D.M., Erickson, J.R., Masor, M.L., Cordle, C.T. and Schaller, J.P. (1998) Modulation of the Immune System by Breast Milk and Infant Formula Containing Nucleotides. Pediatrics, 101, 242-249.

https://doi.org/10.1542/peds.101.2.242

[48] Rajani, P.S., Seppo, A.E. and Jarvinen, K.M. (2018) Immunologically Active Components in Human Milk and Development of Atopic Disease, with Emphasis on Food Allergy, in the Pediatric Population. Frontiers in Pediatrics, 6, 218. https://doi.org/10.3389/fped.2018.00218

[49] Kramer, M.S., Matush, L., Vanilovich, I., Platt, R., Bogdanovich, N. and Sevkovskaya, Z. (2007) Effect of Prolonged and Exclusive Breast Feeding on Risk of Allergy 
and Asthma: Cluster Randomised Trial. BMJ, 335, 815 .

https://doi.org/10.1136/bmj.39304.464016.AE

[50] Kramer, M.S. (1988) Does Breast Feeding Help Protect against Atopic Disease? Biology, Methodology, and a Golden Jubilee of Controversy. The Journal of Pediatrics, 112, 181-190. https://doi.org/10.1016/S0022-3476(88)80054-4

[51] Van Odijk, J., Kull, I., Borres, M.P., Brandtzaeg, P., Edberg, U. and Hanson, L.Å. (2003) Breastfeeding and Allergic Disease: A Multidisciplinary Review of the Literature (1966-2001) on the Mode of Early Feeding in Infancy and Its Impact on Later Atopic Manifestations. Allergy, 58, 833-843. https://doi.org/10.1034/j.1398-9995.2003.00264.x

[52] Greer, F.R., Sicherer, S.H. and Burks, A.W. (2008) Effects of Early Nutritional Interventions on the Development of Atopic Disease in Infants and Children: The Role of Maternal Dietary Restriction, Breastfeeding, Timing of Introduction of Complementary Foods, and Hydrolyzed Formulas. Pediatrics, 121, 183-191. https://doi.org/10.1542/peds.2007-3022

[53] Lodge, C.J., Tan, D.J., Lau, M.X.Z., Dai, X., Tham, R. and Lowe, A.J. (2015) Breastfeeding and Asthma and Allergies: A Systematic Review and Meta-Analysis. Acta Paediatrica, 104, 38-53. https://doi.org/10.1111/apa.13132

[54] Azad, M.B. and Kozyrskyj, A.L. (2012) Perinatal Programming of Asthma: The Role of Gut Microbiota. Clinical and Developmental Immunology, 2012, Article ID: 932072. https://doi.org/10.1155/2012/932072

[55] Bode, L. (2015) The Functional Biology of Breast Milk Oligosaccharides. Early Human Development, 91, 619-622. https://doi.org/10.1016/j.earlhumdev.2015.09.001

[56] Arslanoglu, S., Moro, G.E., Boehm, G., Wienz, F., Stahl, B. and Bertino, E. (2012) Early Neutral Prebiotic Oligosaccharide Supplentation Reduces the Incidence of Some Allergic Manifestations in the First 5 Years of Life. Journal of Biological Regulators and Homeostatic Agents, 26, 49-59.

[57] Castillo-Courtade, L., Han, S., Lee, S., Mian, F.M., Buck, R. and Forsythe, P. (2015) Attenuation of Food Allergy Symptoms Following Treatment with Breast Milk Oligosaccharides in a Mouse Model. Allergy, 70, 1091-1102.

https://doi.org/10.1111/all.12650

[58] Osborn, D.A. and Sinn, J.K. (2013) Prebiotics in Infants for Prevention of Allergy. Cochrane Database of Systematic Reviews, 28, CD006474. https://doi.org/10.1002/14651858.CD006474.pub3

[59] Kull, I., Melen, E., Alm, J., Hallberg, J., Svartengren, M. and van Hage, M. (2010) Breast-Feeding in Relation to Asthma, Lung Function, and Sensitization in Young School Children. Journal of Allergy and Clinical Immunology, 125, 1013-1019. https://doi.org/10.1016/j.jaci.2010.01.051

[60] Pesonen, M., Kallio, M.J.T., Ranki, A. and Siimes, M.A. (2006) Prolonged Exclusive Breastfeeding Is Associated with Increased Atopic Dermatitis: A Prospective Follow-Up Study of Unselected Healthy Newborns from Birth to Age 20 Years. Clinical and Experimental Allergy, 36, 1011-1018. https://doi.org/10.1111/j.1365-2222.2006.02526.x

[61] Hogendorf, A., Stańczyk-Przyłuska, A., Sieniwicz-Luzeńczyk, K., Wiszniewska, M., Arendarczyk, J. and Banasik, M. (2013) Is There Any Association between Secretory IgA and Lactoferrin Concentration in Mature Breast Milk and Food Allergy in Breastfed Children? Developmental Period Medicine, 17, 47-52.

[62] Jarvinen, K.M., Laine, S.T., Jarvenpaa, A.L. and Suomalainen, H.K. (2000) Does Low IgA in Human Milk Predispose the Infant to Development of Cow's Milk 
Allergy? Pediatric Research, 48, 457-462.

https://doi.org/10.1203/00006450-200010000-00007

[63] Saarinen, K.M., Vaarala, O., Klemetti, P. and Savilahti, E. (1999) Transforming Growth Factor- $\beta 1$ in Mothers' Colostrum and Immune Responses to Cows' Milk Proteins in Infants with Cows' Milk Allergy. Journal of Allergy and Clinical Immunology, 104, 1093-1098. https://doi.org/10.1016/S0091-6749(99)70094-1

[64] Thijs, C., Müller, A., Rist, L., Kummeling, I., Snijders, B.E.P. and Huber, M. (2011) Fatty Acids in Breast Milk and Development of Atopic Eczema and Allergic Sensitisation in Infancy. Allergy, 66, 58-67. https://doi.org/10.1111/j.1398-9995.2010.02445.x

[65] Orivuori, L., Loss, G., Roduit, C., Dalphin, J.C., Depner, M. and Genuneit, J. (2014) Soluble Immunoglobulin A in Breast Milk Is Inversely Associated with Atopic Dermatitis at Early Age: The PASTURE Cohort Study. Clinical and Experimental Allergy, 44, 102-112. https://doi.org/10.1111/cea.12199

[66] Ismail, I.H., Licciardi, P.V., Oppedisano, F., Boyle, R.J. and Tang, M.L. (2013) Relationship between Breast Milk sCD 14, TGF- $\beta 1$ and Total IgA in the First Month and Development of Eczema during Infancy. Pediatric Allergy and Immunology, 24, 352-360. https://doi.org/10.1111/pai.12075

[67] Morita, Y., Alberto, C., Jose, E., Yamaide, F., Nakano, T. and Ohnisi, H. (2018) TGF- $\beta$ Concentration in Breast Milk Is Associated with the Development of Eczema in Infants. Frontiers in Pediatrics, 6, 162. https://doi.org/10.3389/fped.2018.00162

[68] Munblit, D., Treneva, M., Peroni, D.G., Colicino, S., Chow, L.Y. and Dissanayeke, S. (2017) Immune Components in Breast Milk Are Associated with Early Infant Immunological Health Outcomes: A Prospective Three-Country Analysis. Nutrients, 9, 532. https://doi.org/10.3390/nu9060532

[69] Cardwell, C.R., Stene, L.C., Ludvigsson, J., Rosenbauer, J., Cinek, O. and Svensson, J. (2012) Breast-Feeding and Childhood-Onset Type 1 Diabetes: A Pooled Analysis of Individual Participant Data from 43 Observational Studies. Diabetes Care, 35, 2215-2225. https://doi.org/10.2337/dc12-0438

[70] Bartz, S. and Freemark, M. (2012) Pathogenesis and Prevention of Type 2 Diabetes: Parental Determinants, Breastfeeding, and Early Childhood Nutrition. Current Diabetes Reports, 12, 82-87. https://doi.org/10.1007/s11892-011-0246-3

[71] Kindgren, E., Fredrikson, M. and Ludvigsson, J. (2017) Early Feeding and Risk of Juvenile Idiopathic Arthritis: A Case Control Study in a Prospective Birth Cohort. Pediatric Rheumatology, 15, 46. https://doi.org/10.1186/s12969-017-0175-Z

[72] Shenoi, S., Shaffer, M.L. and Wallace, C.A. (2016) Environmental Risk Factors and Early-Life Exposures in Juvenile Idiopathic Arthritis: A Case-Control Study. Arthritis Care and Research, 68, 1186-1194. https://doi.org/10.1002/acr.22806

[73] Lionetti, E., Castellaneta, S., Francavilla, R., Pulvirenti, A., Tonutti, E. and Amarri, S. (2014) Introduction of Gluten, HLA Status, and the Risk of Celiac Disease in Children. New England Journal of Medicine, 371, 1295-1303. https://doi.org/10.1056/NEJMoa1400697

[74] van der Sloot, K.W., Amini, M., Peters, V., Dijkstra, G. and Alizadeh, B.Z. (2017) Inflammatory Bowel Diseases: Review of Known Environmental Protective and Risk Factors Involved. Inflammatory Bowel Diseases, 23, 1499-1509. https://doi.org/10.1097/MIB.0000000000001217

[75] Roberts, S.E., Wotton, C.J., Williams, J.G., Griffith, M. and Goldacre, M.J. (2011) Perinatal and Early Life Risk Factors for Inflammatory Bowel Disease. World Journal of Gastroenterology, 17, 743-749. https://doi.org/10.3748/wjg.v17.i6.743 